\title{
Integrated Infrastructure Modelling - Managing Interdependencies with a Generic Approach
}

\author{
Beate R. Dirks, Alexander Otto and Jim W. Hall \\ Environmental Change Institute, University of Oxford, UK
}

\begin{abstract}
Infrastructure provision is a highly challenging task, especially when accounting for climate change mitigation and adaptation needs. Efforts of making infrastructure more efficient and flexible result in an increasing number of sensitive infrastructure interdependencies. This enforces an integrated infrastructure assessment for planning purposes, in contrast to the traditional independent infrastructure-sector modelling.

For the unification of the existing infrastructure-sector models, we propose the implementation of a generic communication interface, which allows the separate sector-models to communicate at the necessarily disaggregate level in order to account for interdependencies appropriately. This approach allows for infrastructure provision modelling under one unified umbrella in a minimally invasive way, while conserving crucial individualities of the separate models. This is achieved through a generic network description, in which we solve the resource allocation through a pragmatic network-flow algorithm that resembles market and consumer behaviour. The developed framework establishes the basis for fully integrated infrastructure evaluation and hence cross-sectorial infrastructure investment decision making - a crucial tool in times of tight governmental budgets.
\end{abstract}

Keywords: Infrastructure Interdependencies, Generic Modelling, Cross-Sectorial Investment Optimization

\begin{abstract}
INTRODUCTION
Infrastructure interdependencies are gaining increased attention due to multiple incidents of catastrophic cascading failures of complex infrastructure networks in recent years ${ }^{1}$. While this forces us to investigate the robustness of the topology of our infrastructure networks, it also sheds light on the increasing relevance of infrastructure interdependencies in all other aspects of infrastructure provision.
\end{abstract}

With the need of climate change mitigation and adaptation we see more efficient and flexible measures of infrastructure provision, to use resources more efficiently and to be able to react to unexpected changes of external driving forces, like climate or global economic trends. Since the behaviour of the socio-economically driven adaptive system of infrastructuresystems is dependent on external driving forces, the choice of service provision might yield widely different amplitudes of the feedbacks between the individual infrastructure systems, depending on changes in the driving forces.

This eradicates the static supply arrangements that traditionally allowed us to account for interdependencies at a highly aggregate level. Instead, we have to account for them at a much more disaggregate level, determined by the dynamics of actual infrastructure provision. The loss of the static situation hence requires more elaborate infrastructure forecasting

1 Eusgeld, I., Nan, C., \& Dietz, S. 'System-of-systems' approach for interdependent critical infrastructures. Reliab. Eng. Syst. Saf. 96(6), 679-686 (2011). Little, R. Controlling cascading failure: understanding the vulnerabilities of interconnected infrastructures. J. Urban Technol. 9(1), 109-123 (2002). 
and planning, moving away from deterministic long-term planning to an increased focus on provision strategies, which do not define the actual investments upfront, but the rules according to which investment decisions are being taken in response to unpredictable changes in the external driving factors.

In times of tight governmental budgets this variability and unpredictability in the planning sphere implies that a crosssectorial optimization of infrastructure investments would be highly valuable to maximize their utility for economic growth and societal wellbeing in general.

In this study we propose a unified generic infrastructure model, drawing upon the existing results on integrated infrastructure investment optimization ${ }^{2}$ and studies on generic description of interdependent infrastructure systems ${ }^{3}$. By casting only the information essentially relevant to infrastructure provision and interdependencies in a generic way, we provide an interface for model-communication at an appropriately disaggregate level, to account for interdependencies. By this we create a generic network-based modelling setting capable of accounting for crucial sector-specific details.

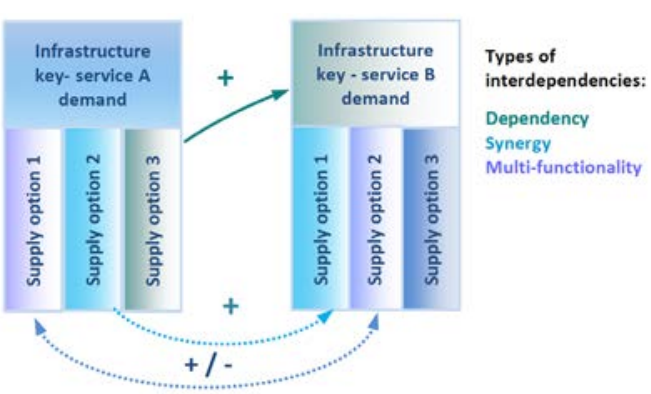

Figure 1. Generic schematics of key-infrastructure systems and their interdependencies. An infrastructure system is serving a key-service demand through supply options. The actual provision of a service can have a positive or negative feedback on either the demand for another infrastructure capacity of another supply option.

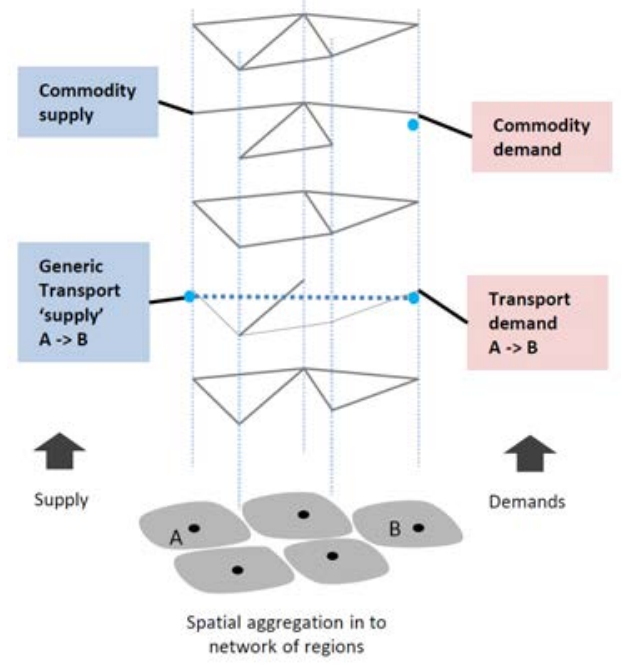

Figure 2. Emergence of the generic network and keyservice classification. Spatial aggregation enables a generic description of the ctate of the system and leads to a natural emergence of a generic network structure. With reference to this generic network, key-services are classified into two types: commodity and transport, depending on whether the respective demands arise local or for a connection.

\section{GENERIC APPROACH}

An integrated infrastructure model has to account for all those infrastructure services that are providing essential, i.e. at a given moment in time irreplaceable, inputs to all that a society does - the so called key-services: energy (in various forms, e.g. electricity, space-heating, fuel etc.), fresh water, transportation, communication and waste treatment (solid waste, sewage, greenhouse gas emissions etc.).

In consultation of previous accounts of infrastructure interdependencies ${ }^{3,4}$, we charac-terise relevant infrastructure interdependencies as visualised in the top of figure 1. A simple dependency is given, if the service provision in one infrastructure system causes a key-service (or network-service) demand in another infrastructure system. In the case of a synergy the provision of one service enables the provision of another service, i.e. increases the respective supply capacity, while in the case of a multi-functionality, the opposite is the case, i.e. the actual service provision decreases the supply capacity of the dependent supply option.

2 Nozick, L.K. et al. Assessing the Performance of Interdependent Infrastructures and Optimizing Investments. Proceedings of the 37th Hawaii International Conference on System Sciences - 2004 C, 1-7 (2004). Ibanez, E. \& McCalley, J.D. Multiobjective evolutionary algorithm for long-term planning of the national energy and transportation systems. Energy Syst. 2(2), 151-169 (2011).

3 Lee, E.E., Mitchell, J.E. \& Wallace, W.a. Restoration of Services in Interdependent Infrastructure Systems: A Network Flows Approach. IEEE Trans. Syst. Man Cybern. Part C 37(6), 1303-1317 (2007). Zhang, P. \& Peeta, S. A generalized modeling framework to analyze interdependencies among infrastructure systems. Transp. Res. Part B 45, 553-579 (2011). 
To account for the relevant interdependencies uniformly, we introduce a generic description of demand and supply as depicted in figure 2, by spatial aggregation over regions, to derive a consistent set of state-of-the-system variables, such like population density, economic activity, as well as the state of the infrastructure systems themselves. This spatial aggregation naturally induces the formation of a generic network of spatial nodes representing regions and of arcs representing connections between regions in infrastructure distribution networks. All information about the underlying system and the infrastructure itself is being accounted for in this setting in respective aggregation and not on the level of individual assets.

Most key-services are fulfilled by the provision of some sort of commodity through a respective transmission network. In these cases the demand for these commodity-type key-services arises locally, irrespective of the origin of the commodity, since the commodity flow units are indistinguishable. In case of transport and communication services we find that the actual key-service is to provide a connection for transportation of some flow good (i.e. passengers, goods or information), between two points in space. Hence, the key-service demand for these transport-type keyservices is a demand for a specific connection.

At each point in time, resources of supply have to be allocated sensibly to prevailing demands. In the case of commodity-type infrastructure systems, the generic network description offers the natural setting to model this task as a network-flow problem. The allocation is hence modelled as a flow of service-units between respective sources and sinks of the generic network, i.e. the commodity generation nodes and the commodity key-service demand nodes.

To include transport-type infrastructure systems into this generic setting, we define artificial transportation generation nodes for each transport demand at the spatial node of the transport-connection origin, as indicated in figure 2. When treating every single transport demand as being only satisfiable by a specific commodity (e.g. personal transport from region $\mathrm{A}$ to $\mathrm{B}$ ), then the allocation of transport services to demands can be modelled as a multi-commodity flow on the respective generic transportation network ${ }^{4}$. This allows us to treat transport type systems identical to commodity type infrastructure networks, under the generalization of allowing for multiple-commodity flows.

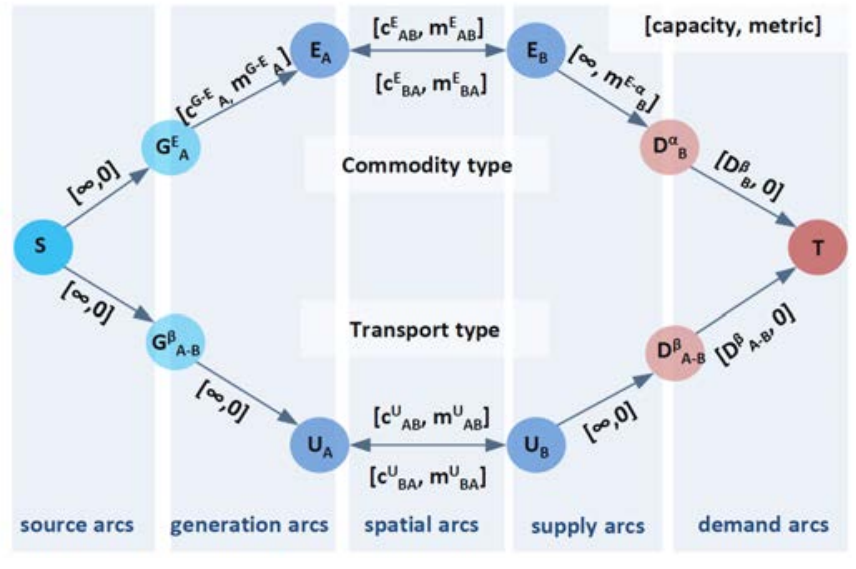

Figure 3. Extended network for the allocation algorithm. S: ultimate source; T: ultimate sink; G: generation; D: demand; A,B: regions; $\partial, B$ : key services; E,U: distribution networks.

\section{SERVICE ALLOCATION AND ACCOUNTING FOR INTERDEPENDENCIES}

The actual service allocation algorithm is being performed on an artificially extended network as shown in figure 3, where generation and transmission assets, as well as demands for key-services are represented through the capacities of suitable edges of the artificial network. To derive an optimal, but yet pragmatic and realistic solution, each edge can have a metric parameter, which is a weighted sum of all relevant supply factors, such as service provision cost and environmental impact. The solution to the allocation problem is found by iteratively optimizing the supply for individual key-service demands and then allocating existing resources in a fair share amongst all demands.

All three forms of interdependencies defined earlier take the generic form in this setting that flows through edges induce changes of capacities in other edges of the extended network. In an outer iteration of the above described algorithm, the interdependency effects are being updated and the flow optimization accordingly refined. Direct dependencies add onto the already existing capacity of demand arcs, while synergies add to the capacities of the edge corresponding to the affected generation or transmission asset. Finally, in the case of multiuse, the capacities of multiple arcs are linked in a way that in each interdependency update, the total shared capacity is allocated to the affected assets in a respective share of relative usage of these edges.

4 Ahuja, R.K. et al. Network flows: Theory, Algorithms and Applications. Prentice Hall (1993) 


\section{CROSS SECTORIAL OPTIMIZATION}

In times of tight governmental budgets and needs of climate change mitigation, we need to be able to optimize infrastructure investments in direct cross-sectorial comparison. Our generic description of infrastructure service generation and allocation allows us to define generic performance indicators.

Straight forward examples include the total metric of the allocation flows, i.e. a measure of how favourable the total solution is. Likewise, the collective demand margin, which is the ratio by which key-service demands can be simultaneously up-scaled and still catered for with supply, is a useful measure to judge the resilience of the overall system to cope with unforeseen demand peaks. Based on such generic performance indicators, investment decisions can then be made in line with generic decision rules, captured in strategies.

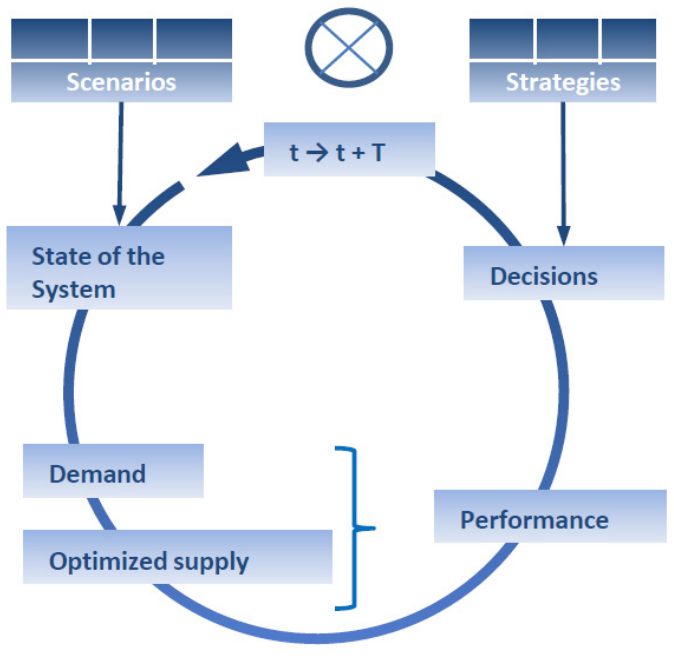

Figure 4. Schematics of the dynamic simulation cycle.

\section{FULLY INTEGRATED SIMULATION SETTING}

Finally, in our fully-integrated infrastructure forecasting model setting the driving forces determining the development of infrastructure provision and key-service demand are subject to uncertainty and hence are creating a set of possible scenarios for the development of key-infrastructure and the availability of supply. The demand for key-services is determined as functions of the state-of-the-system parameters and the external influences captured in the scenarios. The functions determining demand and supply and are highly specific for each infrastructure system and are provided by the individual sectorial models.

With the above described matching algorithm the model setting will then determine the optimized resource allocation in every time-step of the simulation, given as allocation flows of service provision. The performance of the keyinfrastructure system is then a function of initial demand and found allocation flows. These results then feed into the decision-making, which is performed according to the rules captured in a chosen strategy. Finally, an evolutionary step implements the time development of the state-of-the-system, including population and infrastructure ageing, implementation of infrastructure investments, updating of economic performance etc. The simulation of all given strategies under all specified scenarios will result in a performance landscape of strategies and scenarios, which allows to find the 'fittest' strategy of all investigated ones.

\section{CONCLUSION AND OUTLOOK}

In conclusion, we have mapped out a simple, but effective scheme how to cast infrastructure models in order to be able to unify them, allowing to account for interdependencies in the realm of planning purposes and cross-sectorial infrastructure optimization. A case study demonstrating the potential of this framework, when accounting for the impact of interdependencies for planning purposes and hence their consequences for investments, is under way. 